\title{
Influence of five years of antenatal screening on the paediatric cystic fibrosis population in one region
}

\author{
Steven Cunningham, Tom Marshall
}

\begin{abstract}
Background-Antenatal screening for cystic fibrosis has been endorsed by the US National Institutes of Health. Edinburgh is the only city in the UK with an established routine antenatal screening programme for cystic fibrosis.

Aims-To report the change in numbers of infants diagnosed with cystic fibrosis born in Edinburgh after the introduction of antenatal screening for the disease.

Population-Infants diagnosed as having cystic fibrosis (by sweat test or genotyping, or both) in the seven years before antenatal testing (1984-90) and the first five years of antenatal testing (1991-95). Children born in this region who had moved before diagnosis were identified from the UK cystic fibrosis survey database.

Results-The incidence of cystic fibrosis decreased from an average of 4.6 to 1.6 children each year with antenatal screening. The reduction in the incidence $(65 \%)$ was greater than that accounted for by prenatal diagnosis and termination (36\%). Of the eight children born with cystic fibrosis during the period of antenatal screening, five had been subject to antenatal screening: three had only one mutation identified, one was missed due to a laboratory error, and one was identified as a one in four risk, but prenatal diagnosis was not performed.

Conclusions-Antenatal testing for cystic fibrosis has successfully reduced the incidence of cystic fibrosis in this region. Although the numbers are small, it is possible that the reduction in numbers may have been greater than might be expected from antenatal screening alone.

(Arch Dis Child 1998;78:345-348)
\end{abstract}

Department of Child Life and Health,

University of

Edinburgh

S Cunningham

Royal Hospital for Sick Children, Edinburgh T Marshall

Correspondence to: Dr Steven Cunningham, Department of Child Life and Health, University of Edinburgh, 20 Sylvan Place, Edinburgh EH9 1UW.

Accepted 25 November 1997
Keywords: cystic fibrosis; antenatal screening

Cystic fibrosis is the most common inherited disease in the UK to be characterised by chronic disability and early death. The disease is an autosomal recessive inherited disorder with a gene frequency of one in 20 of the general population and a birth incidence of approximately one in 2000 . This combination of high incidence and poor outcome (unlike many other genetically inherited diseases) provides an important opportunity to study the ability of gene testing to reduce the incidence of the disease, the public acceptability of such testing, and the cost of gene screening. Testing the population as a whole would be expensive, both in terms of gene identification and counselling the two million people in the UK with identifiable cystic fibrosis genes. Testing couples attending antenatal clinics reduces the number of subjects requiring screening, identifies the population most interested in their status, and allows prompt counselling and prenatal diagnosis. ${ }^{1}$

Antenatal screening for cystic fibrosis was first started in the larger of Edinburgh's two maternity units (Simpson Memorial Maternity Pavilion) in October 1990 and in the smaller unit (Eastern General Hospital) in January 1992. Screening was offered if antenatal care began before 18 weeks of pregnancy. Initially, samples were taken on a sequential basis (mother first, father if maternal cystic fibrosis gene identified) and then latterly offered to couples (both parents provide a mouthwash sample; the paternal sample is tested only if a maternal mutation is identified). Seventy six per cent of the eligible couples chose to be tested. Screening identified four common cystic fibrosis mutations, which accounted for $85 \%$ of cystic fibrosis mutations in our population (the combination of genes tested has changed with an increased knowledge of gene prevalence in Edinburgh). Couples with two cystic fibrosis mutations were then offered prenatal diagnosis and termination.

Brock has reported the experience of the Edinburgh genetics unit with regard to the number of cystic fibrosis fetuses detected and terminated during the first five years of the antenatal screening programme. ${ }^{2}$ This paper completes the picture with regard to antenatal testing, showing its effect on the incidence of cystic fibrosis in Edinburgh over the past five years and providing details of the cases not identified by antenatal screening.

Neonatal screening is currently available in several regions in the $\mathrm{UK}$ and has been proposed as a nationwide programme. ${ }^{3}$ It is clear, however, that full scale antenatal and neonatal screening programmes operating concurrently in the same region will not be seen as cost effective. Clear, informed decisions are needed about the relative merits of each programme, particularly in view of the recommendation by the US National Institutes of Health to support antenatal, but not neonatal, cystic fibrosis screening. ${ }^{4}$

\section{Methods}

For the period 1984 and 1995 (inclusive), we identified all children who were booked 


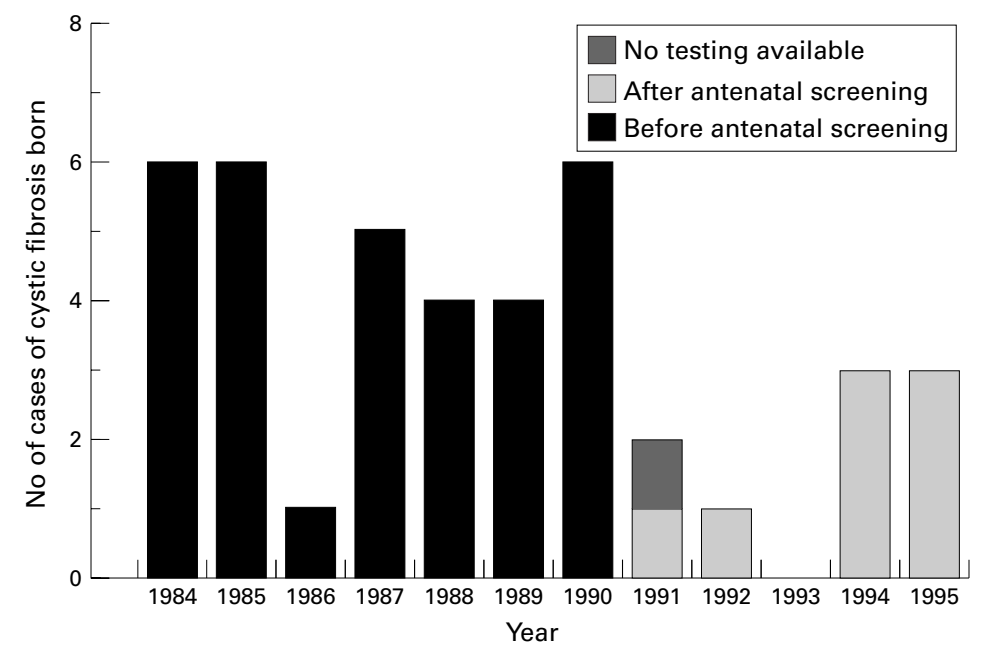

Figure 1 Number of children born with cystic fibrosis before and after the introduction of antenatal screening.

(antenatal visit before or at 18 weeks' gestation) and delivered in an Edinburgh maternity hospital and who were subsequently diagnosed as having cystic fibrosis (confirmed by a sweat test or gene testing, or both). Children cared for in Edinburgh, but booked or born outside the city, have been excluded from this paper. This information was held on the cystic fibrosis database at the Edinburgh Sick Children's NHS Trust (confirmed by parents). The list of names obtained from our database was then checked with details on the UK cystic fibrosis survey database (held in Belfast, UK). The UK cystic fibrosis survey systematically collates data from all UK cystic fibrosis centres on all their patients diagnosed with cystic fibrosis, including the place and date of birth, genotype, and town of residence/review. Children who were born in Edinburgh, but moved away before diagnosis, could thus be identified.

The date of birth of each child was noted and we further assessed those children born after the introduction of antenatal testing at the end of 1990. We obtained details of the antenatal care, antenatal gene testing, and the decisions made in the light of that testing from patient and maternal antenatal hospital notes, and from gene laboratory correspondence held in patient records. The genotype and mode of presentation of those infants not identified antenatally were taken from our cystic fibrosis database. We obtained signed parental consent to discuss the above data for those children who were born after the introduction of antenatal testing.

To ensure that changes in the incidence of cystic fibrosis did not reflect changing demographics, we obtained the number of live births occurring each year in the City of Edinburgh (home and hospital deliveries) from 1984 to 1995, at the General Registrar Office for Scotland. Furthermore, to ensure that the changes in incidence did not simply reflect an alteration in our ability to detect cases over the study period, we assessed the time interval between the initial hospital presentation with clinical features attributable to cystic fibrosis and a diagnosis being made.

\section{Results}

Figure 1 shows the number of children born in Edinburgh each year since 1984 and subsequently diagnosed with cystic fibrosis. Only one child present on the UK cystic fibrosis survey database and born in Edinburgh was not known to us; this child, born before 1991, had moved away from Edinburgh before diagnosis.

An average of 4.6 children each year were diagnosed in the seven years before introducing antenatal gene testing. This average decreased to 1.6 each year after introducing antenatal gene testing (excluding the child born in 1991 at the Eastern General Hospital where gene testing was not available). This represents a reduction in the incidence of cystic fibrosis of $65 \%$.

This reduction has not occurred as a result of changing demographics. The annual birth rate in the City of Edinburgh has fluctuated minimally, with a mean (SD) of 5365 (191) births each year. 1984 and 1995 had the fewest live births over the 11 year period (5090 and 5061 , respectively). Neither does the reduction in incidence reflect a change in our ability to diagnose cystic fibrosis. Most such children are diagnosed at their first hospital presentation with likely symptoms; $61 \%$ before the introduction of antenatal gene testing and $88 \%$ after the introduction of antenatal gene testing (not including two infants identified antenatally).

Eight children born in the City of Edinburgh after the introduction of antenatal testing have subsequently been diagnosed as having cystic fibrosis (table 1). In three cases antenatal testing was not performed. In two cases diagnosis was through the routine genetic diagnostic

Table 1 Details of the eight infants born with cystic fibrosis during the first five years of the antenatal screening programme

\begin{tabular}{|c|c|c|c|}
\hline Case No & Age at diagnosis & $\Delta$ genotype & Antenatal details \\
\hline 1 & Prenatal & $\Delta \mathrm{F} 508 / \Delta \mathrm{F} 508$ & $\begin{array}{l}\text { Paternal mutation already known. Maternal antenatal diagnostic } \\
\text { laboratory diagnosis (close family history) }\end{array}$ \\
\hline 2 & Birth (meconium ileus) & N1303K/Q493X & $\begin{array}{l}\text { Previous genetic counselling (close family history). Not screened in } \\
\text { pregnancy }\end{array}$ \\
\hline 3 & 20 months. Persistent right upper lobe collapse & $\Delta \mathrm{F} 508 / \mathrm{P} 67 \mathrm{~L}$ & $\begin{array}{l}\text { Donor insemination. P67L mutation not part of screen performed on } \\
\text { donor. No maternal antenatal screen }\end{array}$ \\
\hline 4 & 2 months. Failure to thrive & $\Delta \mathrm{F} 508 / 1717-1 \mathrm{G}-\mathrm{A}$ & $\begin{array}{l}\text { Antenatal screening. 1717-1G-A mutation not part of screen. } \\
\text { Unavoidable miss }\end{array}$ \\
\hline 5 & 6 weeks. Persistent respiratory syncytial virus bronchiolitis & $\Delta \mathrm{F} 508 / ?$ & $\begin{array}{l}\text { Antenatal screening. Paternal mutation still not identified. } \\
\text { Unavoidable miss }\end{array}$ \\
\hline 6 & Birth (meconium ileus) & $\Delta \mathrm{F} 508 / 1717-1 \mathrm{G}-\mathrm{A}$ & $\begin{array}{l}\text { Antenatal screening. 1717-1G-A mutation not part of screen. } \\
\text { Unavoidable miss }\end{array}$ \\
\hline 7 & 23 months. Pseudomonas chest infection & $\Delta \mathrm{F} 508 / \Delta \mathrm{F} 508$ & Antenatal screening. Laboratory error. Avoidable miss \\
\hline 8 & Prenatal one in four risk & $\Delta \mathrm{F} 508 / \mathrm{R} 117 \mathrm{H}$ & $\begin{array}{l}\text { Antenatal screening identified one in four risk. Twin pregnancy; } \\
\text { prenatal diagnosis not requested }\end{array}$ \\
\hline
\end{tabular}


laboratory because of cystic fibrosis in a first degree family member of one of the parents. In case 1 a paternal $\Delta \mathrm{F} 508$ was known before pregnancy and a maternal $\Delta \mathrm{F} 508$ was detected on antenatal testing (the parents wished to continue the pregnancy); in case 2 prepregnancy genetic testing and counselling did not detect a Q493X mutation in one parent. Case 3 was a donor insemination birth; the semen donor was screened for four cystic fibrosis mutations at the time of donation, but P67L was not part of that screen. The child's mother was not screened antenatally because of donor screening.

In five cases antenatal screening was performed. In three cases $(4,5$, and 6$)$, antenatal screening detected only one of the two cystic fibrosis mutations present in the couple; in two cases the unidentified mutation was 1717$1 \mathrm{G}-\mathrm{A}$ (considered to account for $<1 \%$ of cystic fibrosis mutations in our population); in the other no mutation has been identified. These missed cases were unavoidable with current mutation screening methods. In case 7 , laboratory error did not detect a $\Delta \mathrm{F} 508$ mutation in a maternal sample; the paternal sample was therefore not analysed. Case 8 was antenatally screened and identified as a one in four risk. This mother did not wish prenatal diagnosis in view of the risks to her twin pregnancy.

\section{Discussion}

Our experience is that this introduction of antenatal screening has been followed by a $65 \%$ reduction in the number of cases of cystic fibrosis diagnosed in children. Antenatal screening identifies the genes responsible for $85 \%$ of mutations for cystic fibrosis in our community. Detecting $85 \%$ in each partner produces a homozygous detection rate of $72 \%$. As screening was performed for $70 \%$ of antenatal couples, we expected to identify $50 \%$ of high risk cystic fibrosis pregnancies (a one in four risk). If all these patients accepted prenatal diagnosis, then this would detect $50 \%$ of cases each year, so reducing the liveborn incidence of cystic fibrosis to 2.3 cases each year. Brock reported that 22 high risk (one in four) couples were identified in the period reported and all eight couples with a positive prenatal diagnosis accepted termination, giving a termination rate of 1.7 each year ${ }^{2}$ - that is, a $36 \%$ reduction at 4.7 births each year. As antenatal screening accounts for only $36 \%$ of our observed $65 \%$ reduction in the incidence of cystic fibrosis, other factors may be involved. Another region performing selective antenatal screening noted a decrease in the incidence of cystic fibrosis that was not completely explained by the number of terminations performed. $^{5}$

Natural disease variability might account for the decreasing incidence of cystic fibrosis in our region after the introduction of gene testing; however, we do not consider that this would account for such a prolonged period of low incidence in an area with a stable birth population. Late presentation may also account for the discrepancy in the incidence. The children reported here are 1-6 years old (an

\section{Key messages}

- Antenatal screening for cystic fibrosis has produced an effective reduction in the number of new cases

- The reduction in the number of new cases of cystic fibrosis was greater than that expected from terminations after prenatal diagnosis

- The reasons for the greater than expected decrease in the number of cases of cystic fibrosis may represent a change in reproductive behaviour by couples identified as carriers

- The effective detection rate of antenatal screening would be improved if acceptance levels for antenatal screening programmes were improved

average of about 3.5 years). In the years 198490 , the median age at diagnosis in our region was 5 months, with $88 \%$ of cases diagnosed before 3.5 years. At the current rates we can therefore expect one further child born in the period $1991-5$ to present in the future. In fact, we may not even see late presenters at this rate because antenatal screening has identified $\mathrm{R} 117 \mathrm{H}$, which is a late presenting genotype with a mean age of presentation of 10.4 years. $^{6}$ The reduced incidence may also represent antenatal screening reducing the numbers of affected siblings born before the diagnosis of a subsequent child; this has been reported as accounting for $11-20 \%$ of cystic fibrosis diagnoses in areas without antenatal testing. ${ }^{78}$ Finally, it is possible that antenatal screening has influenced the reproductive behaviour of those couples identified as high risk (22 couples in 4.7 years), particularly those with previously healthy children. Ninety per cent of parents of children with cystic fibrosis would find antenatal diagnosis acceptable in the event of a future pregnancy, though only $20-50 \%$ would proceed to the termination of an affected fetus. ${ }^{910}$ The dilemma posed to parents in terminating an affected fetus when they already have a child with the disease prompts $60-77 \%$ to decide against having further children. ${ }^{511} 12$ The Edinburgh genetics group have shown that carriers identified by antenatal screening (one in 20 of the screened population) did not report an intention to alter their reproductive behaviour. ${ }^{13}$ The intentions of the 22 high risk couples identified were not given in that report. Four of the 22 high risk couples identified in the first five years returned for further prenatal diagnosis during the five year period (DJH Brock, personal communication).

Two methods of screening are available. Neonatal screening is performed in six regions in the UK and is proposed as a nationwide scheme, ${ }^{3}$ whereas antenatal screening is only routinely performed in Edinburgh. A panel convened by the US National Institutes of Health has recommended that cystic fibrosis gene testing should be offered to pregnant women and couples currently planning a preg- 
nancy. The panel did not recommend mass screening of newborn infants as they concluded it had no proved clinical or financial benefit. ${ }^{4}$

Neonatal screening has been assessed in some regions and a two or three staged screening programme is now proposed nationwide. This involves immune reactive trypsin measurement from a heelprick sample, followed by the identification of cystic fibrosis mutations, and then a sweat test to confirm the diagnosis. ${ }^{3}$ Early neonatal diagnosis may help to reduce early tissue damage, morbidity, and increase life expectancy. ${ }^{14-16}$ Neonatal screening would identify $70 \%$ of undiagnosed cases within 10 weeks of birth $^{17}$; the test has a false negative rate of $5-8 \%,{ }^{18}$ would be too late in identifying those infants who present early (about $18 \%$ present with meconium ileus ${ }^{17}$ ), and may miss those genotypes with borderline sweat tests in the first few years of life. ${ }^{19} \mathrm{New}$ born screening also requires a number of healthy children to undergo sweat testing, with associated parental anxiety. ${ }^{20}$

Antenatal diagnosis provides parents with a choice of termination in their first pregnancy. As all fetuses diagnosed with cystic fibrosis in our cohort were terminated, the service appears to be one parents find acceptable and useful. Unfortunately, impact success in the antenatal testing programme, particularly in terms of cost, has been assessed by the number of affected pregnancies terminated. ${ }^{21}$ We are disappointed that, in the Edinburgh cohort, $24 \%$ of parents did not wish to be tested, and over $60 \%$ of those stated their reason as opposition to termination or that they considered the risk of cystic fibrosis too low. ${ }^{22}$ The effective detection rate of antenatal screening (that is, the number of cases terminated plus those identifiable at birth) could be increased if couples who decline testing on the grounds of opposition to termination could be informed of the possible advantages of early diagnosis without undue pressure for prenatal diagnosis and termination. The National Childbirth Trust has highlighted the problem of parents' discomfort with perceived pressure for termination when antenatal diagnosis is offered. ${ }^{23}$ If such parents accepted antenatal screening, then the effective detection rate of both neonatal and prenatal screening programmes would be similar at around $70 \%$. Selective neonatal screening could be considered as a further option in the $6.4 \%$ of pregnancies with only one mutation detected antenatally. However, as this would diagnose only one case in every 164 single mutation pregnancies, the benefit would have to be balanced against the parental anxiety evoked.

Our thanks to Joan Smith, Aileen Mallinson, and the UK cystic fibrosis survey for their assistance with data collection. Thanks lso to Professor DJH Brock for his assistance in confirming genetic data.

1 Livingstone J, Axton RA, Gilfillan A, et al. Antenatal screening for cystic fibrosis; a trial of the couple model. BMF 1994;308:1459-62.

2 Brock DJH. Prenatal screening for cystic fibrosis; 5 years' experience reviewed. Lancet 1996;347:148-50.

3 Littlewood JM. Why newborn screening for cystic fibrosis is clinically and cost effective, and now a priority in cystic fibrosis care. London: Cystic Fibrosis Trust (United Kingdom) care. London: Cystic

4 Macready N. US endorses testing for cystic fibrosis in pregnant women. BMf 1997;314:1299.

5 Green MR, Weaver LT, Heeley AF, et al. Cystic fibrosis identified by neonatal screening; incidence, genotype, and early natural history. Arch Dis Child 1993;68:464-7.

6 The Cystic Fibrosis Genotype-Phenotype Consortium. Correlation between genotype and phenotype in patients with cystic fibrosis. N Engl F Med 1993;329:1308-13.

7 MacLusky IB, Canny GS, Levison H. Cystic fibrosis. Pediatric Reviews and Communications I 1987:343-89.

8 FitzSimmons SC. The changing epidemiology of cystic fibrosis. F Pediatr 1993;122:1-9.

9 Wertz DC, Rosenfield JM, Janes SR, Erbe RW. Attitudes toward abortion among parents of children with cystic fibrosis. Am 7 Public Health 1991;81:992-6.

10 Al-Jadar LN, Goodchild MC, Ryley HC, Harper PS. Attitudes of parents of cystic fibrosis children towards neonatal screening and antenatal diagnosis. Clin Genet 1990;38:460-5.

11 Dankert-Roelse JE, Te Meerman GJ, Knol K, Ten Kate LP. Effect of screening for cystic fibrosis on the influence of genetic counselling. Clin Genet 1987;32:271-5.

12 Braekeleer M, Murray J, Daigneault J, Allard C, Simard F, Aubin G. Disease knowledge and reproductive attitudes of parents having a child with cystic fibrosis. Ann Genet 1994; 37:89-92.

13 Axworthy D, Brock DJH, Bobrow M, Marteau TM. Psychological impact of population based carrier testing for cystic fibrosis; 3 year follow up. Lancet 1996;347:1443-6.

14 Khan TZ, Wagener JS, Bost T, Martinez J, Accurso FJ, Riches DWH. Early pulmonary inflammation in infants with cystic fibrosis. Am $\mathcal{F}$ Respir Crit Care Med 1995;151: 1075-82.

15 Dankert-Roelse JE, Te Meerman GJ. Long term prognosis of patients with cystic fibrosis in relation to early detection by neonatal screening and treatment in a cystic fibrosis centre. Thorax 1995;50:712-18.

16 Dankert-Roelse JE, Te Meerman GJ, Martijn A, Ten Kate LP, Knol K. Survival and clinical outcome in patients with cystic fibrosis, with or without neonatal screening. F Pediatr 1989;114:362-7.

17 Phelan PD. Neonatal screening for cystic fibrosis. Thorax 1995;50:705-6.

18 Ranieri E, Lewis BD, Gerace RL, et al. Neonatal screening for cystic fibrosis using immunoreactive trypsinogen and direct gene analysis: four years experience. BMF 1994;308: 1469-72.

19 Fitzgerald D, Van Asperen P, Henry R, et al. Delayed diagnosis of cystic fibrosis in children with a rare genotype

20 Chatfield S, Owen G, Ryley HC, et al. Neonatal screening for cystic fibrosis in Wales and West Midlands; clinical assessment after five years of screening. Arch Dis Child assessment after

21 Cuckle HS, Richardson GA, Sheldon TA, Quirke P. Cost effectiveness of antenatal screening for cystic fibrosis. BMF 1995;311:1460-3.

22 Mennie ME, Gilfillan A, Compton ME, Liston WA, Brock DJH. Prenatal cystic fibrosis carrier screening; factors in a woman's decision to decline testing. Prenat Diagn 1993;13: $807-14$

23 Dodds $\mathrm{R}$. The stress of test in pregnancy; an antenatal screening survey. London: National Childbirth Trust (UK), 1997. 